\title{
A NEW CONSTRUCTION OF WAVELET SETS
}

\author{
EUGEN J. IONASCU
}

\begin{abstract}
We show that the class of (dyadic) wavelet sets is in one-to-one correspondence to a special class of Lebesgue measurable isomorphisms of $[0,1)$ which we call wavelet induced maps. We then define two natural classes of maps $\mathcal{W I}_{1}$ and $\mathcal{W I}_{2}$ which, in order to simplify their construction, retain only part of the characterization properties of a wavelet induced map. We prove that each wavelet induced map appears from the Schröder-Cantor-Bernstein construction applied to some $u \in \mathcal{W I}_{1}$ and $v \in \mathcal{W I}_{2}$. Consequently, the construction of a wavelet set is basically equivalent to the easier construction of two maps $u \in \mathcal{W I}_{1}$ and $v \in \mathcal{W I}_{2}$. Some older results on wavelet sets are recovered using this new point of view. The connectivity result of Speegle ([20]) is recaptured and the completeness in the natural metric of the class of wavelet sets is reestablished. Although these ideas seem to generalize to more than one dimension, specific examples are given only in the one dimensional case.
\end{abstract}

\section{INTRODUCTION}

In 9] the authors introduced the notion of wavelet set which turned out to be one of the building blocks of their approach to wavelet analysis from an operator theory point of view. At the same time, and independently, the notion of wavelet set appeared as the support set of so called MSF-wavelets (minimally supported frequency) in a series of papers: 12, 14] and [15].

One easy way to fabricate a wavelet is to normalize the Fourier transform of the characteristic function of a wavelet set. Wavelet sets have been generalized to $n$ dimensions (see [10] and [11]). The important result of the existence of wavelets for unitary systems having an expansive dilation matrix was based on the existence of wavelet sets. These ideas were taken into the realm of frame theory and the notion of wavelet set was generalized even further to frame (tight frame or normalized tight frame) wavelet sets in [4, [5], [6]-[8]. In [3] the authors give an ingenious description of how one can construct a wavelet set. The purpose of this paper is to consider a different approach to the construction of (dyadic) wavelet sets which is purely set theoretic.

Date: August 30th, 2002.

1991 Mathematics Subject Classification. Primary 42C40, 42C30; Secondary 03E15.

Key words and phrases. wavelet set, wavelet induced isomorphism, Schröder-Cantor-Bernstein construction. 


\section{Preliminary Results}

We denote by $\mu$ the Lebesgue measure on $\mathbb{R}$. The $L^{2}$-space with respect to $\mu$ will be written simply as $L^{2}(\mathbb{R})$. An orthonormal wavelet is (cf. [9]) a function $w \in L^{2}(\mathbb{R})$ for which the family of functions $\left\{w_{j, k}\right\}_{j, k \in \mathbb{Z}}$ defined by

$$
w_{j, k}(s)=2^{j / 2} w\left(2^{j} s-k\right), \quad s \in \mathbb{R}, j, k \in \mathbb{Z},
$$

is an orthonormal basis for $L^{2}(\mathbb{R})$.

We say that a measurable subset $W$ of $\mathbb{R}$ is a wavelet set if $\frac{1}{\sqrt{\mu(W)}} \chi_{W}=\widehat{w}$, where $w$ is a wavelet in $L^{2}(\mathbb{R})$ and $\widehat{w}$ is the Fourier-Plancherel transform on $L^{2}(\mathbb{R})$ of the function $w$ and which for $f \in L^{1}(\mathbb{R}) \cap L^{2}(\mathbb{R})$ is defined by

$$
\widehat{f}(x)=\frac{1}{\sqrt{2 \pi}} \int_{\mathbb{R}} e^{-i t x} f(t) d \mu(t), \quad x \in \mathbb{R} .
$$

One of the simplest examples of wavelet sets is the Littlewood-Paley wavelet set $E:=$ $[-2 \pi,-\pi) \cup[\pi, 2 \pi)$. A less obvious example is the following union of eight intervals

$$
S:=\begin{aligned}
& {\left[-\frac{4 \pi}{3},-\frac{5 \pi}{4}\right) \cup\left[-\pi,-\frac{2 \pi}{3}\right) \cup\left[-\frac{5 \pi}{8},-\frac{\pi}{2}\right) \cup\left[\frac{4 \pi}{7}, \frac{2 \pi}{3}\right)} \\
& \cup\left[\frac{3 \pi}{4}, \pi\right) \cup\left[\frac{4 \pi}{3}, \frac{11 \pi}{8}\right) \cup\left[4 \pi, \frac{32 \pi}{7}\right) \cup\left[\frac{11 \pi}{2}, 6 \pi\right) .
\end{aligned}
$$

The next result was announced independently in [12] and 9] and it is definitely the first step in a better understanding of the notion of a wavelet set. We refer the reader to [16] for a proof of this proposition. In order to state the result let us introduce some notation. Let $\tau: \mathbb{R} \rightarrow E$ be the function defined by $\tau(x)=x+2 j \pi$, where $j$ is the unique integer satisfying $x+2 j \pi \in E$ and let $\delta: \mathbb{R} \backslash\{0\} \rightarrow E$ be the map defined by $\delta(x)=2^{k} x$, where $k$ is the unique integer for which $2^{k} x \in E$.

Proposition 2.1. The following conditions are equivalent for any measurable subset $W$ of $\mathbb{R}$ :

(i) $W$ is a wavelet set,

(ii) there exists a set $W^{\prime}$ such that $W^{\prime}=W$ a.e., the family of sets $\left\{W^{\prime}+2 k \pi\right\}_{k \in \mathbb{Z}}$ is a partition of $\mathbb{R}$ and, at the same time, the family $\left\{2^{k} W^{\prime}\right\}_{k \in \mathbb{Z}}$ is a partition of $\mathbb{R} \backslash\{0\}$,

(iii) there exists a set $W^{\prime \prime}$ such that $W^{\prime \prime}=W$ a.e. and $\tau_{\mid W^{\prime \prime}}, \delta_{\mid W^{\prime \prime}}: W^{\prime \prime} \rightarrow E$ are measurable bijections.

In [16] a wavelet set having the property of $W^{\prime}$ in (ii) (or equivalently the property of $W^{\prime \prime}$ in (iii)) was called regularized. Let us denote by $\mathcal{W S}$ the class of all wavelet sets. 
The class $\mathcal{W S}$ is very rich. In [18] it was shown that every point $x_{0} \in \mathbb{R} \backslash\{0\}$ contains a neighborhood which is a part of a wavelet set. In 20] it was proved that $\mathcal{W S}$ is pathconnected (in the norm topology on $L^{2}(\mathbb{R})$ when $\mathcal{W S}$ is naturally imbedded in $L^{2}(\mathbb{R})$ ). It was shown in 13] that $\mathcal{W S}$ becomes a complete metric space $(\mathcal{W S}, d)$ with the metric:

$$
d\left(W_{1}, W_{2}\right):=\mu\left(W_{1} \nabla W_{2}\right)^{\frac{1}{2}}+\left(\int_{W_{2} \nabla W_{1}} \frac{1}{|x|} d \mu(x)\right)^{\frac{1}{2}},
$$

where $W_{1} \nabla W_{2}=\left(W_{1} \backslash W_{2}\right) \cup\left(W_{2} \backslash W_{1}\right)$. In spite of this richness, it is not very obvious how would one construct a wavelet set.

Thus by part (iii) of this proposition, we can associate with every $W \in \mathcal{W S}$ a measurable bijection on $E$ defined by

$$
h_{W}:=\tau_{\mid W^{\prime}} \circ\left(\delta_{\mid W^{\prime}}\right)^{-1} .
$$

By its definition, this map is essentially uniquely determined by $W$ in the sense that for two sets $W^{\prime}$ and $W^{\prime \prime}$ as in Proposition 2.1, the maps $h_{W^{\prime}}, h_{W^{\prime \prime}}$ coincide almost everywhere $[\mu]$. Thus we will denote this map simply by $h_{W}$. It turns out that the conjugation $\widetilde{h}_{W}:=$ $\xi \circ h_{W} \circ \xi^{-1}:[0,1) \rightarrow[0,1)$ of $h_{W}$, by the function $\xi: E \rightarrow[0,1)$ defined by

$$
\xi(x)=\left\{\begin{array}{l}
\frac{x}{2 \pi}, \quad x \in[\pi, 2 \pi), \\
\frac{x}{2 \pi}+1, \quad x \in[-2 \pi,-\pi),
\end{array}\right.
$$

takes a simpler form than $h_{W}$.

DeFinition 2.2. For every wavelet set $W$ the map $\widetilde{h}_{W}$ constructed as above is called an wavelet induced isomorphisms of $[0,1)$.

We have the following characterization of the class of wavelet sets in terms of the corresponding maps $\widetilde{h}_{W}$.

Proposition 2.3. Let $W \in \mathcal{W S}$ and $\widetilde{h}_{W}$ be defined as above. Then the map $\widetilde{h}_{W}$ has the following properties:

(i) $\widetilde{h}_{W}$ is a measurable bijection of $[0,1)$,

(ii) there exists a measurable partition $\left\{A_{k}\right\}_{k \in \mathbb{Z}}$ of $\left[\frac{1}{2}, 1\right)$ and a measurable partition $\left\{B_{k}\right\}_{k \in \mathbb{Z}}$ of $\left[0, \frac{1}{2}\right)$, such that

$$
\widetilde{h}_{W}(x)=\left\{\begin{array}{l}
\left\lfloor 2^{k} x\right\rfloor, \quad x \in A_{k}, k \in \mathbb{Z}, \\
\left\lfloor 2^{k}(x-1)\right\rfloor, \quad x \in B_{k}, k \in \mathbb{Z},
\end{array}\right.
$$

where $\lfloor x\rfloor$ denotes the fractional part of the real number $x$. 
(iii) if $h$ is a map satisfying $(i)$ and (ii) then there exists a wavelet set $W$ such $h=\widetilde{h}_{W}$;

(iv) for two wavelet sets $W_{1}$ and $W_{2}$ we have $W_{1} \cap W_{2}=\delta_{\mid W_{1}}^{-1} \circ \xi^{-1}(\Omega)=\delta_{\mid W_{2}}^{-1} \circ \xi^{-1}(\Omega)$, a.e. $[\mu]$, where $\Omega=\left\{x \in[0,1): \widetilde{h}_{W_{1}}(x)=\widetilde{h}_{W_{2}}(x)\right\}$.

Proof. One can easily check that $\tau(t)=\xi^{-1}\left(\left\lfloor\frac{t}{2 \pi}\right\rfloor\right)$ for every $t \in \mathbb{R}$. First, let us observe that if $x \in\left[\frac{1}{2}, 1\right)$ then $u=\xi^{-1}(x)=2 \pi x$ and let us write $\delta^{-1}(u)=2^{k} u$ with $k \in \mathbb{Z}$. Hence, using the formula mentioned above, we have $\tau\left(2^{k} u\right)=\xi^{-1}\left(\left\lfloor\frac{2^{k} u}{2 \pi}\right\rfloor\right)=\xi^{-1}\left(\left\lfloor 2^{k} x\right\rfloor\right)$. Thus $\widetilde{h}_{W}(x)=\xi\left(\tau_{\mid W}\left(2^{k} u\right)\right)=\left\lfloor 2^{k} x\right\rfloor$.

If $x \in[0,1 / 2)$, then $u=\xi^{-1}(x)=2 \pi(x-1)$ and if we write $\delta^{-1}(u)=2^{k} u$ with $k \in \mathbb{Z}$, we get $\widetilde{h}_{W}(x)=\xi\left(\tau_{\mid W}\left(2^{k} u\right)\right)=\left\lfloor\frac{2^{k} u}{2 \pi}\right\rfloor=\left\lfloor 2^{k}(x-1)\right\rfloor$. This proves claim (ii) of the proposition.

To prove claim (iii), let us consider that $h$ has the properties $(i)$ and $(i i)$ and we denote $h_{1}=\xi^{-1} \circ h \circ \xi: E \rightarrow E$. Because of (6)], we obtain that for every $x \in E$ there exist $k(x), l(x) \in \mathbb{Z}$ such that $h_{1}(x)=2^{k(x)} x+2 l(x) \pi$. By the assumptions on $h$ the maps $x \rightarrow k(x), x \rightarrow l(x)$ are measurable. Then we define $\psi: E \rightarrow \mathbb{R}$ by $\psi(x)=2^{k(x)} x, x \in E$, and $W:=\psi(E)$. Clearly, $W$ is a measurable set, $\psi$ is one-to-one and $\left(\delta_{\mid W}\right)^{-1}=\psi$. Finally we define $\varphi: W \rightarrow E$ by $\varphi(y)=y+2 l\left(\psi^{-1}(y)\right) \pi$. Then one can check that $h_{1}=\varphi \circ \psi$ and $\varphi(y)=\tau_{\mid W}(y), y \in W$. Since $h_{1}$ is one-to-one and $\psi$ is onto we conclude that $\varphi$ is one-to-one. Also, $\varphi$ is onto since $h_{1}$ is. By Proposition 2.1 we see that $W$ is a wavelet set. According to (4) and using the above facts it follows that $h_{W}=h_{1}$ and so $\widetilde{h}_{W}=h$.

To prove (iv) let us show the equality in question by double inclusion. For $y \in W_{1} \cap W_{2}$ we have $s:=\delta_{\mid W_{1}}(y)=\delta_{\mid W_{2}}(y)=\delta(y)$. Then $y=\delta_{\mid W_{1}}^{-1}(s)=\delta_{\mid W_{2}}^{-1}(s)$. Let us denote $\xi(s)$ by $u$. In other words $s=\xi^{-1}(u)$. This lets us write $y=\delta_{\mid W_{1}}^{-1} \circ \xi^{-1}(u)=\delta_{\mid W_{2}}^{-1} \circ \xi^{-1}(u)$. This means that we need to check that $u \in\left\{x \in[0,1): \widetilde{h}_{W_{1}}(x)=\widetilde{h}_{W_{2}}(x)\right\}$ or equivalently $u \in\left\{\xi(t): t \in E, \quad h_{W_{1}}(t)=h_{W_{2}}(t)\right\}$. Since $u=\xi(s)$ we need to see why is it true that $h_{W_{1}}(s)=h_{W_{2}}(s)$. Using (4) this last equality is the same as $\tau_{\mid W_{1}}(y)=\tau_{\mid W_{2}}(y)$ which is true. This argument shows that $W_{1} \cap W_{2} \subset \delta_{\mid W_{1}}^{-1} \circ \xi^{-1}(\Omega)$ and $W_{1} \cap W_{2} \subset \delta_{\mid W_{2}}^{-1} \circ \xi^{-1}(\Omega)$.

For the opposite inclusion let us start with $y=\delta_{\mid W_{1}}^{-1} \circ \xi^{-1}(u)$ where $u$ satisfies $\widetilde{h}_{W_{1}}(u)=$ $\widetilde{h}_{W_{2}}(u)$. As before we let $s:=\xi^{-1}(u)$. This implies $h_{W_{1}}(s)=h_{W_{2}}(s)$ or $\tau_{\mid W_{1}} \circ \delta_{\mid W_{1}}^{-1}(s)=$ $\tau_{\mid W_{2}} \circ \delta_{\mid W_{2}}^{-1}(s)$. Taking in account that $\tau\left(t_{1}\right)=\tau\left(t_{2}\right)$ implies $t_{1}=t_{2}+2 k \pi$ for some $k \in \mathbf{Z}$ we obtain that $\delta_{\mid W_{1}}^{-1}(s)=\delta_{\mid W_{2}}^{-1}(s)+2 k \pi, k \in \mathbf{Z}$. Let $\delta_{\mid W_{1}}^{-1}(s)=2^{n} s$ and $\delta_{\mid W_{2}}^{-1}(s)=2^{m} s$, for some $m$ and $n \in \mathbf{Z}$. If $n \neq m$ then $s=\frac{2 k \pi}{2^{n}-2^{m}}$. It is clear that the set

$$
\mathcal{F}=\left\{\frac{2 l \pi}{2^{i}-2^{j}}: i, j, l \in \mathbf{Z}, \mathbf{i} \neq \mathbf{j}\right\}
$$


is countable and so it has measure zero. Assuming that $s \notin \mathcal{F}$, we get $n=m$ and $k=0$ which implies $y=\delta_{\mid W_{1}}^{-1} \circ \xi^{-1}(u)=\delta_{\mid W_{1}}^{-1}(s)=\delta_{\mid W_{2}}^{-1}(s) \in W_{1} \cap W_{2}$. This argument proves that $\delta_{\mid W_{1}}^{-1} \circ \xi^{-1}(\Omega) \backslash \mathcal{F} \subset W_{1} \cap W_{2}$ and $\delta_{\mid W_{2}}^{-1} \circ \xi^{-1}(\Omega) \backslash \mathcal{F} \subset W_{1} \cap W_{2}$.

For the wavelet set $S$ defined by (2), we computed the map $\widetilde{h}_{S}$ and obtained

$$
\widetilde{h}_{S}(x)=\left\{\begin{array}{l}
\left\lfloor 2^{-1}(x-1)\right\rfloor \text { on } \quad\left[0, \frac{1}{3}\right) \cup\left[\frac{3}{8}, \frac{1}{2}\right), \\
\left\lfloor 2^{2} x\right\rfloor \text { on }\left[\frac{1}{2}, \frac{4}{7}\right) \cup\left[\frac{11}{16}, \frac{3}{4}\right), \\
\left\lfloor 2^{-1} x\right\rfloor \text { on }\left[\frac{4}{7}, \frac{2}{3}\right) \cup\left[\frac{3}{4}, 1\right), \\
\lfloor x\rfloor \text { on }\left[\frac{1}{3}, \frac{3}{8}\right) \cup\left[\frac{2}{3}, \frac{11}{16}\right) .
\end{array}\right.
$$

One can check that this map is a measurable bijection from $[0,1)$ into $[0,1)$. The graph of it is on page 6 .

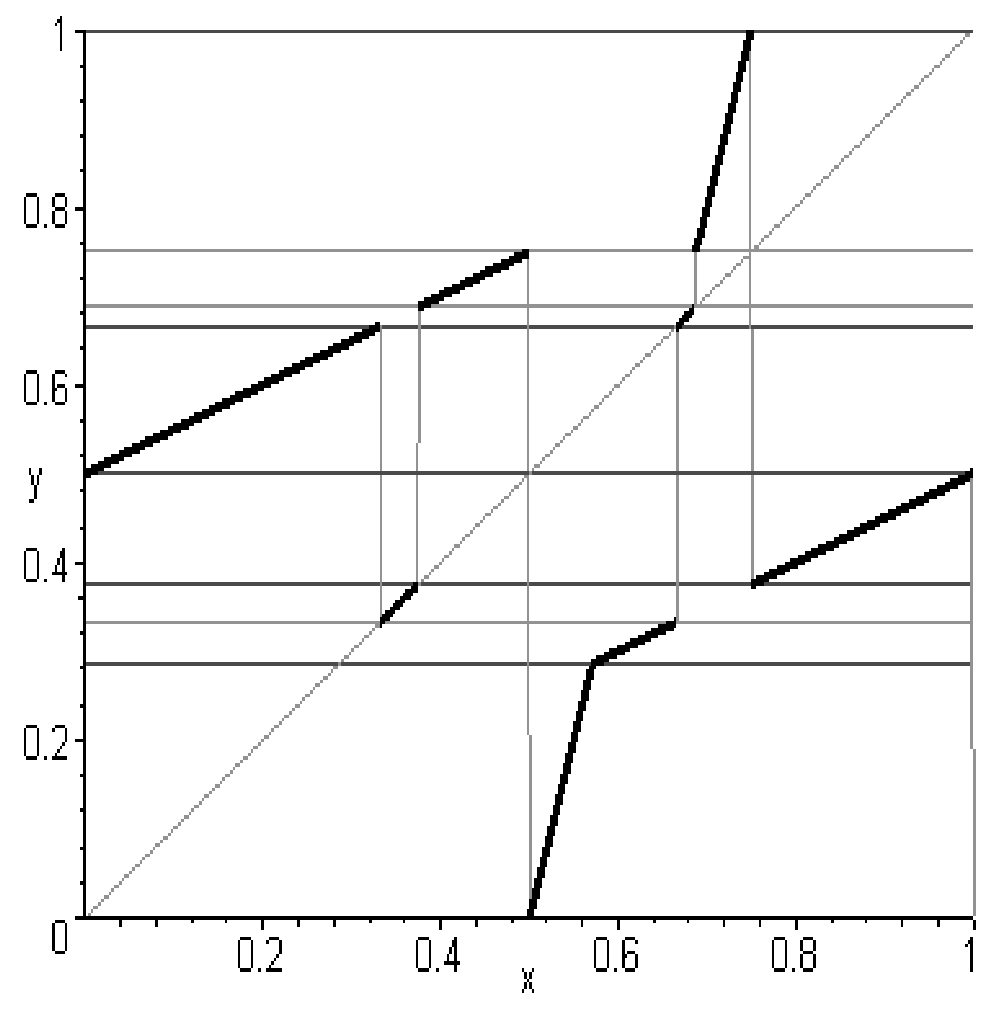

We denote the class of all wavelet induced isomorphisms by $\mathcal{W I}$. The wavelet induced map for and wavelet set played an important role in the series of papers [1], [2] and [16]. 
One essential hypothesis that we needed in these works was the existence of a measurable cross section for the isomorphism (i.e. a measurable set which contains exactly one point from each orbit of the isomorphism). This existence was partially solved in [1] but it is still an open conjecture in the general situation. In this paper we have no need for such an assumption. However, we obtain a similar path of wavelets in Theorem 3.5] as in [16. By Proposition 2.3 every map in $\mathcal{W I}$ can be expressed as in (6).

\section{The SchröDer-Cantor-Bernstein CONStruction}

Let $\mathcal{W I}_{1}$ be the class of all measurable maps $f:[0,1) \rightarrow[0,1)$ that are defined by a measurable partition $\left\{A_{k}\right\}_{k \in \mathbb{N}}$ of $\left[\frac{1}{2}, 1\right)$ and a measurable partition $\left\{B_{k}\right\}_{k \in \mathbb{N}}$ of $\left[0, \frac{1}{2}\right)$, such that

$$
f(x)=\left\{\begin{array}{l}
\frac{x}{2^{k}}, \quad x \in A_{k}, \quad k \geq 1, \\
\frac{x-1}{2^{k}}+1, \quad x \in B_{k}, \quad k \geq 1 .
\end{array}\right.
$$

The following is a simple consequence of the above definition.

LEMMA 3.1. Every function $f \in \mathcal{W I}_{1}$ is one-to-one and $\mu(f(\sigma)) \leq \frac{1}{2} \mu(\sigma)$ for every measurable subset $\sigma$ of $[0,1)$.

Proof. Let $x_{1}, x_{2} \in[0,1), x_{1} \neq x_{2}$. We need to analyze essentially four cases. If $x_{1}, x_{2} \in A_{k}$ or $x_{1}, x_{2} \in B_{k}$ for some $k \geq 1$ clearly $f\left(x_{1}\right) \neq f\left(x_{2}\right)$. If $x_{1} \in A_{k}$ and $x_{2} \in B_{l}$ for some $k, l \geq 1$ then $f\left(x_{2}\right)=\left(x_{2}-1\right) / 2^{l}+1 \geq 1 / 2>f\left(x_{1}\right)$. Suppose now $x_{1} \in A_{k}$ and $x_{2} \in A_{l}$ for $k>l \geq 1$. In this case $x_{1}, x_{2} \geq 1 / 2$ and so $f\left(x_{2}\right)=x_{2} / 2^{l} \geq 1 / 2^{l+1} \geq 1 / 2^{k}>f\left(x_{1}\right)$. Finally, if $x_{1} \in B_{k}$ and $x_{2} \in B_{l}$ for some $k>l \geq 1$ we have $x_{1}, x_{2} \in[0,1 / 2)$. Hence, $f\left(x_{2}\right)=\left(x_{2}-1\right) / 2^{l}+1<1-1 / 2^{l+1} \leq 1-1 / 2^{k} \leq f\left(x_{1}\right)$.

For the second part of this lemma let us observe that we can write $\sigma$ as a disjoint union $\sigma=\bigcup_{n \geq 1} \sigma_{n} \cup \bigcup_{n \geq 1} \sigma_{n}^{\prime}$ where $\sigma_{n}=\sigma \cap A_{n}, \sigma_{n}^{\prime}=\sigma \cap B_{n}, n \in \mathbb{N}$. Clearly, $\mu\left(f\left(\sigma_{n}\right)\right) \leq$ $(1 / 2) \mu\left(\sigma_{n}\right)$ and $\mu\left(f\left(\sigma_{n}^{\prime}\right)\right) \leq(1 / 2) \mu\left(\sigma_{n}^{\prime}\right)$ for all $n \in \mathbb{N}$. Adding up all these inequalities we obtain that $\mu(f(\sigma)) \leq \mu(\sigma)$ for every measurable set $\sigma$.

Remark. Let us emphasize the fact that constructing a map $f$ in $\mathcal{W I}_{1}$ is just a simple matter of choosing two measurable partitions: one for $[0,1 / 2)$ and one for $[1 / 2,1)$. The next figure shows the line segments of equations involved in (9) and gives an idea of why Lemma 3.1 is true. 


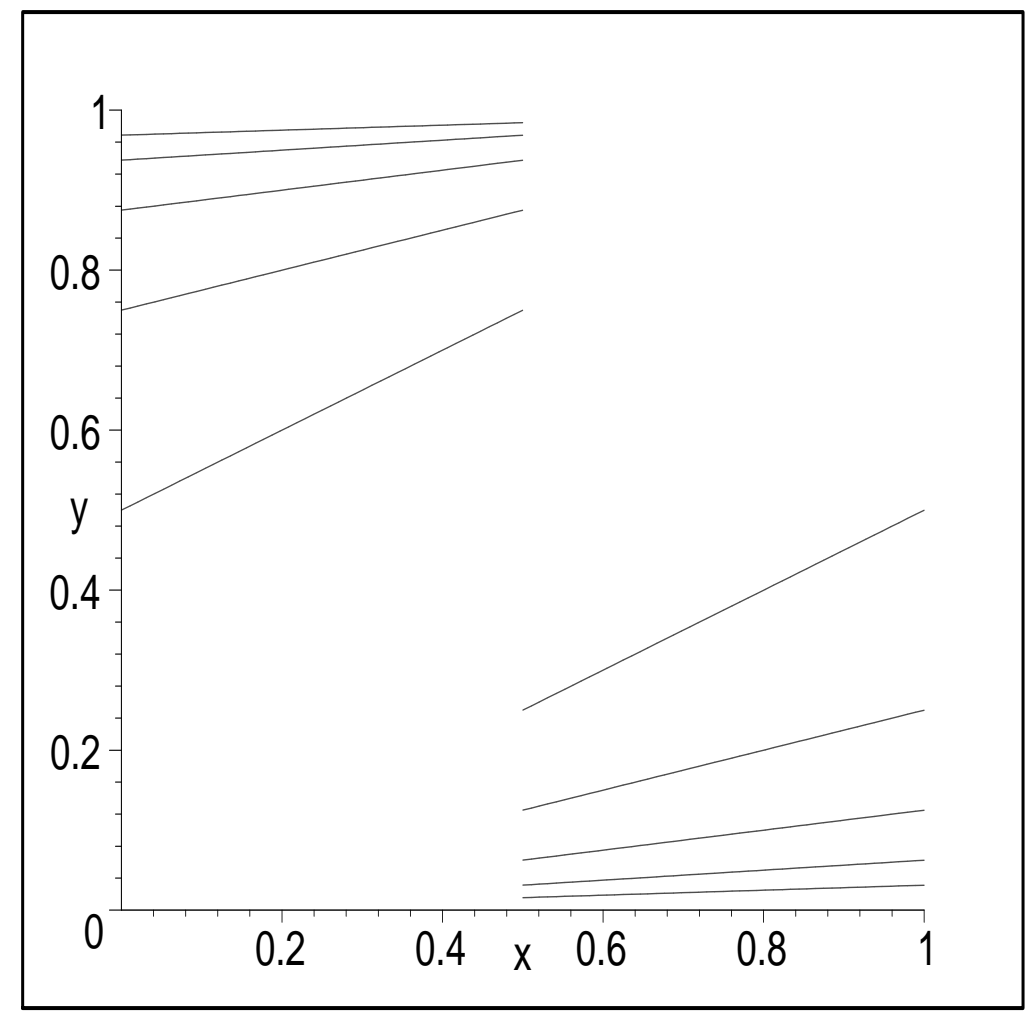

We introduce now the class $\mathcal{W I}_{2}$ of all measurable one-to-one maps $g:[0,1) \rightarrow[0,1)$ so that for every $x \in[0,1)$ there exist $k, l \in \mathbb{Z}, k, l \geq 0$ such that $g(x)=\frac{x+l}{2^{k}}$. We remind the reader the following fact from set theory known as the Schröder-Cantor-Bernstein theorem.

Proposition 3.2. (Schröder-Cantor-Bernstein) Let $A$ and $B$ two arbitrary sets, $u: A \rightarrow B$ and $v: B \rightarrow A$ be two one-to-one maps. Then the map $u \diamond v: A \rightarrow B$ defined by

$$
(u \diamond v)(x)=\left\{\begin{array}{l}
u(x) \quad \text { for } \quad x \in \bigcup_{k=0}^{\infty}(v \circ u)^{k}(A \backslash v(B)), \\
v^{-1}(x) \quad \text { for } \quad x \in \bigcup_{k=0}^{\infty}(v \circ u)^{k}(v(B) \backslash(v \circ u)(A)) \cup \bigcap_{k=0}^{\infty}(v \circ u)^{k}(A),
\end{array}\right.
$$

is a bijection.

Remark. It is easy to see that the inverse function of $u \diamond v$ given as in (10) is in fact $v \diamond u$. We are now ready for the main result of this note. This next theorem has almost the flavor of a factorization theorem.

THEOREM 3.3. Every wavelet induced isomorphism $h$ is the result of the SchröderCantor-Bernstein construction, i.e. $h=u \diamond v$ a.e. for some $u \in \mathcal{W I}_{1}$ and $v \in \mathcal{W I}_{2}$ where $\diamond$ 
is defined in (10). Conversely, every map $u \diamond v$ with $u \in \mathcal{W I}_{1}$ and $v \in \mathcal{W I}_{2}$ is an wavelet induced isomorphism. (The writing $h=u \diamond v$ is in general not unique.)

Proof. The last part of the theorem follows from the Proposition 2.2 and the fact that $u \diamond v$ is by construction a measurable bijection of the form (ii) in Proposition 2.2. To show the first part, let us start with $h \in \mathcal{W} \mathcal{I}$ and define $\mathcal{D}_{1}$ be the measurable set of all $x \in[0,1)$ for which the $k$ in the definition (6) is a negative integer. We let $u(x)=h(x)$ for $x \in \mathcal{D}_{1}$ and extend $u$ to $\mathcal{D}_{2}=[0,1) \backslash \mathcal{D}_{1}$ such that $u \in \mathcal{W I}_{1}$. This extension is not unique but it can be easily constructed as observed in the remark following the Lemma 3.1. (For instance, for the map given in (8) we can take

$$
u(x)=\left\{\begin{array}{l}
\frac{x-1}{2}+1 \text { on }\left[0, \frac{1}{2}\right), \\
\frac{x}{2} \text { on }\left[\frac{1}{2}, 1\right),
\end{array}\right.
$$

in which case $\mathcal{D}_{1}=\left[0, \frac{1}{3}\right) \cup\left[\frac{3}{8}, \frac{1}{2}\right) \cup\left[\frac{4}{7}, \frac{2}{3}\right) \cup\left[\frac{3}{4}, 1\right)$.) To continue the proof, let $\mathcal{R}_{1}:=u\left(\mathcal{D}_{1}\right)$ and $\mathcal{R}_{2}:=[0,1) \backslash \mathcal{R}_{1}$. Next we define $v$ on $\mathcal{R}_{2}$ as $v(x)=h^{-1}(x)\left(x \in \mathcal{R}_{2}\right)$. Let us observe that $v$ is defined in accordance to the properties of the maps in $\mathcal{W I}_{2}$. We will show next that $v$ can be extended to $[0,1)$ in such a way that $v \in \mathcal{W I}_{2}$. (In the case of the map defined by (6) we take

$$
v(x)= \begin{cases}\frac{x+3}{4} & \text { on } \quad\left[0, \frac{1}{4}\right), \\ \frac{x+1}{2} & \text { on } \quad\left[\frac{1}{4}, \frac{1}{2}\right) \cup\left[\frac{5}{8}, \frac{3}{4}\right), \\ x & \text { on } \quad\left[\frac{1}{2}, \frac{5}{8}\right) \cup\left[\frac{7}{8}, 1\right), \\ \frac{x}{2} \text { on } \quad\left[\frac{3}{4}, \frac{7}{8}\right)\end{cases}
$$

which is just one of the various possible extentions.)

Getting back to the general situation, it turns out that independently of what extensions one might consider for $u$ and $v$, the map $u \diamond v$ constructed as in (10) is the same as $h$ a.e. The following lemma solves the existence of the extension $v$.

LemmA 3.4. The map $v: \mathcal{R}_{2} \rightarrow[0,1)$ defined by $v(x)=h^{-1}(x)\left(x \in \mathcal{R}_{2}\right)$ can be extended to a map in $\mathcal{W I}_{2}$. 
ProOF. Let us define the extension inductively in the following way. First we just pick a bijection $\varphi: \mathbb{N} \rightarrow\{(k, l): k, l \in \mathbb{N} \cup\{0\}\}$. For $n \in \mathbb{N}$ we denote by $\varphi_{1}(n)$ [resp. $\left.\varphi_{2}(n)\right]$ the first component [resp. second component] of $\varphi(n)$. Then the initial step is to extend $v$ to $E_{1}:=\mathcal{R}_{2} \cup F_{1}$ where $F_{1}:=\left\{x \in[0,1) \backslash \mathcal{R}_{2}:\left(x+\varphi_{1}(1)\right) / 2^{\varphi_{2}(1)} \in[0,1) \backslash v\left(\mathcal{R}_{2}\right)\right\}$. (Clearly $F_{1}$ may be empty.) In any case we define $v_{1}(x)=\left(x+\varphi_{1}(1)\right) / 2^{\varphi_{2}(1)}$ for all $x \in F_{1}$ and $v_{1}(x)=v(x)$ for $x \in \mathcal{R}_{2}$. Suppose we defined $v_{n}$ on $E_{n}:=E_{n-1} \cup F_{n}$ as an extension of $v_{n-1}$. Let $v_{n+1}$ be the extension of $v_{n}$ to $E_{n+1}:=E_{n} \cup F_{n+1}$ where $F_{n+1}:=\left\{x \in[0,1) \backslash E_{n}\right.$ : $\left.\left(x+\varphi_{1}(n+1)\right) / 2^{\varphi_{2}(n+1)} \in[0,1) \backslash v_{n}\left(E_{n}\right)\right\}$ defined as $v_{n+1}(x)=\left(x+\varphi_{1}(n+1)\right) / 2^{\varphi_{2}(n+1)}$ for all $x \in F_{n+1}$. By the way these extensions are constructed it is easy to see that each $v_{n}$ is a one-to-one map. If for some $n \in \mathbb{N}$ we have $E_{n}=[0,1)$ a.e., then the proof is finished since $v_{n}$ would be the extension we were looking for.

We may assume then that $\mu\left([0,1) \backslash E_{n}\right)>0$ for every $n \in \mathbb{N}$. In this case we let $E_{\infty}=\cup_{n=1}^{\infty} E_{n}$ and define the extension $\widetilde{v}$ of $v$ to $E_{\infty}$ in the usual way: $\widetilde{v}(x)=v_{n}(x)$ if $x \in E_{n}$. Because $v_{n+1}$ is an extension of $v_{n}$ the map $\widetilde{v}$ is well defined. We claim that $E_{\infty}=[0,1)$ a.e. in which case then $\widetilde{v} \in \mathcal{W I}_{2}$ is the extension we want.

In order to prove this claim we proceed by way of contradiction and assume that $\mu(U)>0$ where $U:=[0,1) \backslash E_{\infty}$. One can easily show, using similar arguments to those in the proof of Lemma 3.1. that $\mu\left(\widetilde{v}\left(E_{\infty}\right)\right) \leq \mu\left(E_{\infty}\right)$. This shows that $\mu(V)>0$ where $V:=[0,1) \backslash \widetilde{v}\left(E_{\infty}\right)$. Obviously, $U$ and $V$ are measurable sets. It is known (see [19]) that the transformation $T(x)=\lfloor 2 x\rfloor$ is an ergodic transformation on $[0,1)$ with respect to an invariant measure which is equivalent to Lebesgue measure. This implies that $\mu\left(T^{k}(V) \cap U\right)>0$ for some $k \in \mathbb{N}$. Equivalently we have $\mu\left(\left(2^{k} V-l\right) \cap U\right)>0$ for some $l \in \mathbb{N} \cup\{0\}$. Let us write $\widetilde{U}$ for the set $\left(2^{k} V-l\right) \cap U$. We have $(x+l) / 2^{k} \in V$ for every $x \in \widetilde{U}$. If we let $n:=\varphi^{-1}(k, l)$ it follows that $\widetilde{U} \subset E_{n} \cap U$ which contradicts the fact that $E_{n} \cap U=\emptyset\left(E_{n} \subset E_{\infty}=[0,1) \backslash U\right)$.

Returning to the proof of the Theorem 3.3 let $u \in \mathcal{W I}_{1}$ and $v \in \mathcal{W I}_{2}$ be the maps constructed as above. We need to show that $u \diamond v=h$ almost everywhere. Since $\mathcal{R}_{1}:=u\left(\mathcal{D}_{1}\right)$ and $u_{\mid \mathcal{D}_{1}}=h_{\mid \mathcal{D}_{1}}$ it follows that $h^{-1}\left(\mathcal{R}_{1}\right)=\mathcal{D}_{1}$ and $h^{-1}\left(\mathcal{R}_{2}\right)=\mathcal{D}_{2}$ since $h$ is one-to-one. Hence, $v\left(\mathcal{R}_{2}\right)=h^{-1}\left(\mathcal{R}_{2}\right)=\mathcal{D}_{2}$. Since $u$ and $v$ are one-to-one maps, it follows that $u\left(\mathcal{D}_{2}\right) \subset \mathcal{R}_{2}$ and $v\left(\mathcal{R}_{1}\right) \subset \mathcal{D}_{1}$. Let $\mathcal{S}=[0,1) \backslash v([0,1))$. Clearly $\mathcal{S} \subset \mathcal{D}_{1}$. Thus, $u(\mathcal{S}) \subset \mathcal{R}_{1}$ and then $(v \circ u)(\mathcal{S}) \subset \mathcal{D}_{1}$. Inductively it follows that all the sets $(v \circ u)^{k}(\mathcal{S}), k \in \mathbf{N} \cup \mathbf{0}$, are contained in $\mathcal{D}_{1}$. Therefore for $x \in \bigcup_{k \geq 0}(v \circ u)^{k}(\mathcal{S})$ according to (10) we have $u \circ v(x)=u(x)=h(x)$.

Let $\mathcal{N}=v([0,1)) \backslash(v \circ u)([0,1))$. Clearly $\mathcal{N} \subset \mathcal{R}_{2}$ and inductively it follows that all the sets $(v \circ u)^{k}(\mathcal{N}) \subset \mathcal{R}_{2}$. Thus, for $x \in \bigcup_{k \geq 0}(v \circ u)^{k}(\mathcal{N})$ we have $u \diamond v(x)=v^{-1}(x)=$ $\left(h^{-1}\right)^{-1}(x)=h(x)$. 
Using Lemma 3.1 we observe that the set $\bigcap_{k \geq 0}(v \circ u)^{k}([0,1))$ must have Lebesgue measure zero. Hence $u \diamond v=h$ almost everywhere.

Remark. If $v=i d$ then $u \diamond v=i d$. We will use this fact to obtain as a corollary Speegle's result in [20]. The convergence seems to be in a stronger metric but in fact the two metrics are equivalent on the class of wavelets.

THeOREM 3.5. For every two wavelet sets $W_{0}$ and $W_{1}$ one can find a chain of wavelet sets $\left\{W_{t}\right\}_{t \in[0,1]}$ connecting them and such that $d\left(W_{t}, W_{s}\right) \rightarrow 0$ if $t \rightarrow s$ where the metric $d$ is given by (圆).

Proof. It is obvious that we just need to consider the case $W_{1}=E$ (the LittlewoodPaley wavelet set). Denote $W_{0}$ simply by $W$ and let $h:=h_{W}$ be the wavelet induced isomorphism of $W$. According to the Theorem 3.3 we can find $u \in \mathcal{W I}_{1}$ and $v \in \mathcal{W I}_{2}$ such that $u \diamond v=h$ almost everywhere. The idea of our proof is to connect $v$ with $i d$ by a continuous chain of maps in $\mathcal{W I}_{2}$ and then use the second part of Theorem 3.3 to construct $h_{t}=u \diamond v_{t}$. Then we just take $W_{t}$ the corresponding wavelet set to $h_{t}$.

Lemma 3.6. There exist a chain $\left\{v_{t}\right\}_{t \in[0,1]}$ such that $v_{0}=v, v_{1}=i d$ and $\mu\left(\left\{x: v_{t}(x) \neq\right.\right.$ $\left.\left.v_{s}(x)\right\}\right) \rightarrow 0$ as $t \rightarrow s$.

Proof. We may assume without loss of generality that $v$ is not the identity function. Hence there must exist a measurable set $U \subset[0,1)$ such that $\mu(v(U))<\mu(U)$. Since $\mu(v(L)) \leq \mu(L)$ for every measurable subset of $[0,1)$ we see that the set $V=[0,1) \backslash v([0,1))$ has positive Lebesgue measure. We define what is going to be the first part of our chain by $v_{t}^{1}(x)=v(x)$ if $x<t$ and $x \in[0,1) \backslash V$ or $x \geq t$ and $v_{t}^{1}(x)=x$ if $x<t$ and $x \in V$. Clearly if $0 \leq t<s \leq 1$ then $\left\{x: v_{t}^{1}(x) \neq v_{s}^{1}(x)\right\} \subset[t, s]$ and therefore $\mu\left(\left\{x: v_{t}^{1}(x) \neq\right.\right.$ $\left.v_{s}^{1}(x)\right\} \rightarrow 0$ as $t \rightarrow s$. Notice that the maps $v_{t}^{1}$ are by construction one-to-one and then automatically $v_{t}^{1} \in \mathcal{W I}_{2}$ for all $t$. The map $v_{1}^{1}$ is $i d$ on $V$ and $v$ everywhere else. Notice that $[0,1) \backslash\left(v_{1}^{1}([0,1))=v(V)\right.$. Let us denote by $\mathcal{Z}$ the set of fixed points of $v$. We have clearly $V \cap \mathcal{Z}=\emptyset$ and therefore $v^{k}(V) \cap \mathcal{Z}=\emptyset$ for all $k \in \mathbf{N}$. We observe that $[0,1)$ can be partitioned as $[0,1)=\bigcup_{k \geq 0} v^{k}(V) \cup \bigcap_{k \geq 0} v^{k}([0,1))$. Using the above observations $\mathcal{Z} \subset \bigcap_{k \geq 0} v^{k}([0,1))$ and since $\mu(v(L)) \leq \frac{\mu(L)}{2}$ for every measurable set contained in $[0,1) \backslash \mathcal{Z}$ we conclude that $\bigcap_{k \geq 0} v^{k}([0,1)) \backslash \mathcal{Z}$ has Lebesgue measure zero. Hence to finish the proof we will extend the chain $v_{t}^{1}$ with a chain $v_{t}^{2}$ which will connect $v_{1}^{1}$ to the $v_{1}^{2}$ which is $i d$ on $V \cup v(V)$. So, the next chain is defined by $v_{t}^{2}(x)=v_{1}^{1}(x)$ if $x<t$ and $x \in[0,1) \backslash v(V)$ or $x \geq t$ and $v_{t}^{2}(x)=x$ if $x<t$ and $x \in v(V)$. Observe that maps $v_{t}^{2}$ are one-to-one and so they 
are in $\mathcal{W I}_{2}$. As before the continuity of $\left\{v_{t}^{2}\right\}_{t}$ is insured. The map $v_{1}^{2}$ is $i d$ on $V \cup v(V)$ and $v$ everywhere else. Then we continue inductively constructing $v_{t}^{3}, v_{t}^{4}, \ldots$, in a similar manner. To end the proof we put these countably many chains together in an obvious way to form the required chain. More precisely, we scale and glue the chains $\left\{v_{t}^{1}\right\},\left\{v_{t}^{2}\right\},\left\{v_{t}^{3}\right\}, \ldots$ to create the final chain $\left\{v_{t}\right\}$ where $v_{1}=i d$ (we allocate an interval of length $1 / 2$ for $\left\{v_{t}^{1}\right\}$, an interval of length $1 / 4$ for $\left\{v_{t}^{2}\right\}$ and so on). It is easy to see that the continuity claim still holds, i.e., that $\mu\left(\left\{x: v_{t}(x) \neq v_{s}(x)\right\}\right) \rightarrow 0$ as $t \rightarrow s$.

Returning to the proof of Theorem 3.5 we want to establish next the equivalent of the distance given by (3) at the level of $\mathcal{W} \mathcal{I}$.

LEMMA 3.7. Let $h_{1}$ and $h_{2}$ be the wavelet induced isomorphisms associated with two wavelet sets $W_{1}$ and $W_{2}$ then the metric given by (3) satisfies:

$$
\left.\left.d\left(W_{1}, W_{2}\right)=\left(4 \pi \mu\left(\omega^{\prime}\right)\right)^{1 / 2}+(2 \nu(\omega)\}\right)\right)^{1 / 2},
$$

where the measure $\nu$ on $[0,1)$ is given by $d \nu(x)=\frac{1}{1-x} \chi_{[0,1 / 2)}(x) d \mu(x)+\frac{1}{x} \chi_{[1 / 2,1)}(x) d \mu(x)$ and $\omega^{\prime}:=\left\{x \in[0,1): h_{1}^{-1}(x) \neq h_{2}^{-1}(x)\right\}$ and $\omega:=\left\{x \in[0,1): h_{1}(x) \neq h_{2}(x)\right\}$.

Proof. In order to establish (13) we observe that the property (iv) in Proposition 2.3 implies that $W_{1} \nabla W_{2}=\left(\delta_{\mid W_{1}}^{-1} \circ \xi^{-1}([0,1)) \backslash \delta_{\mid W_{1}}^{-1} \circ \xi^{-1}(\Omega)\right) \bigcup\left(\delta_{\mid W_{2}}^{-1} \circ \xi^{-1}([0,1)) \backslash \delta_{\mid W_{1}}^{-1} \circ \xi^{-1}(\Omega)\right)$ (disjoint union). Hence

$$
\begin{aligned}
\mu\left(W_{1} \nabla W_{2}\right)= & \mu\left(\delta_{\mid W_{1}}^{-1} \circ \xi^{-1}([0,1) \backslash \Omega)\right)+\mu\left(\delta_{\mid W_{2}}^{-1} \circ \xi^{-1}([0,1)) \backslash \Omega\right)= \\
& \mu\left(\delta_{\mid W_{1}}^{-1} \circ \xi^{-1}(\omega)\right)+\mu\left(\delta_{\mid W_{2}}^{-1} \circ \xi^{-1}(\omega)\right) .
\end{aligned}
$$

Taking in account that $\mu$ is invariant under translations and homogeneous under dilations, i.e., $\mu(t U)=t \mu(U)$ for every measurable set $U$ and every positive real number $t$, we obtain

$$
\begin{gathered}
\mu\left(W_{1} \nabla W_{2}\right)=\mu\left(\tau_{\mid W_{1}} \circ \delta_{\mid W_{1}}^{-1} \circ \xi^{-1}(\omega)\right)+\mu\left(\tau_{\mid W_{2}} \circ \delta_{\mid W_{2}}^{-1} \circ \xi^{-1}(\omega)\right)= \\
2 \pi \mu\left(\xi \circ \tau_{\mid W_{1}} \circ \delta_{\mid W_{1}}^{-1} \circ \xi^{-1}(\omega)\right)+2 \pi \mu\left(\xi \circ \tau_{\mid W_{2}} \circ \delta_{\mid W_{2}}^{-1} \circ \xi^{-1}(\omega)\right)= \\
2 \pi \mu\left(h_{1}(\omega)\right)+2 \pi \mu\left(h_{2}(\omega)\right) .
\end{gathered}
$$

Let us observe that $h_{1}(\omega)=h_{2}(\omega)=\omega^{\prime}$ and so $h^{-1}\left(\omega^{\prime}\right)=h^{-1}\left(\omega^{\prime}\right)=\omega$. Indeed, $h_{1}(\omega)=$ $h_{1}([0,1) \backslash \Omega)=[0,1) \backslash h_{1}(\Omega)$ and one can easily check that $h_{1}(\Omega)=\Omega^{\prime}$ where $\Omega^{\prime}=\{x$ : $\left.h_{1}^{-1}(x)=h_{2}^{-1}(x)\right\}$ where for the convenience of the reader we recall that $\Omega=\{x \in[0,1)$ : $\left.h_{1}(x)=h_{2}(x)\right\}$. As a result, (14) becomes

$$
\mu\left(W_{1} \nabla W_{2}\right)=4 \pi \mu\left(\omega^{\prime}\right) .
$$

This equality allows us to get the first term in (13). In order to obtain the second part of (13) we begin by considering the measure on $\mathbb{R} \backslash\{0\}$ defined as $d \lambda(x)=\frac{1}{|x|} d \mu(x)$. As before 
we have

$$
\lambda\left(W_{1} \nabla W_{2}\right)=\lambda\left(\delta_{\mid W_{1}}^{-1} \circ \xi^{-1}(\omega)\right)+\lambda\left(\delta_{\mid W_{2}}^{-1} \circ \xi^{-1}(\omega)\right) .
$$

But $\lambda$ is invariant under dilations. Hence the above changes into:

$$
\lambda\left(W_{1} \nabla W_{2}\right)=2 \lambda\left(\xi^{-1}(\omega)\right) .
$$

One can easily check that $\lambda \circ \xi^{-1}=\nu$. Putting (15), (16), and (3) together we obtain (13).

Returning now to the proof of Theorem 3.5 let us define $\left\{h_{t}\right\}_{t \in[0,1]}$ by $h_{t}:=u \diamond v_{t}$, with $v_{t}$ given by Lemma 3.6. According to Theorem [3.3] and Proposition [2.3] each $h_{t}$ is an wavelet induced isomorphism which corresponds to some wavelet $W_{t}$. Because $h_{0}=u \diamond v$ and $h_{1}=u \diamond i d$ we have $W_{0}=W$ and $W_{1}=E$. We need to introduce one more notation: for two maps $f$ and $g$ having the same domain of definition let $\omega(f, g)$ be defined by

$$
\omega(f, g)=\{x: f(x) \neq g(x)\}) .
$$

By the way $\left\{v_{t}\right\}$ was constructed if $0 \leq t<s \leq 1$ then $\omega\left(v_{t}, v_{s}\right)$ is contained in an interval of length less than $s-t$. By the definition (10) we see that $\omega\left(h_{t}, h_{s}\right)$ is contained in a set of Lebesgue measure less than $2\left[(t-s) / 2+(t-s) / 4+\ldots+(t-s) / 2^{k}+\ldots\right]=2(t-s)$ because $u$ is "measure contractive" is the sense of Lemma 3.1. Therefore $\mu\left(\omega\left(h_{t}, h_{s}\right)\right) \rightarrow 0$ as $t \rightarrow s$ or $s \rightarrow t$. To finish the proof we observe that $\nu$ is equivalent with the Lebesgue measure on $[0,1)$ and $h_{t}^{-1}=v_{t} \circ u$. Using the same arguments as above we have $\mu\left(\omega\left(h_{t}^{-1}, h_{s}^{-1}\right)\right) \rightarrow 0$ as $t \rightarrow s$ or $s \rightarrow t$. Finally we use Lemma 3.7 to end the proof.

Remark. The Theorem 3.5 has the advantage of being a more constructive result than the one in [20]. We observe also that by construction $W_{t} \subset W \cup E$ for every $t$. This construction is in some sense very similar to the one given in [16] where only a partial result was obtained.

THEOREM 3.8 (Garrigos-Speegle[13]). The class $\mathcal{W S}$ is complete in the metric given by (3).

ProOF. Let us start with a sequence of wavelet set $\left\{W_{n}\right\}$ which is Cauchy in the metric in (3). Let us consider their corresponding wavelet induced isomorphisms $\left\{h_{n}\right\}_{n \in \mathbf{N}}$. According to Lemma 3.7. we have $\mu\left(\omega\left(h_{n}, h_{m}\right)\right) \rightarrow 0$ and $\mu\left(\omega\left(h_{n}^{-1}, h_{m}^{-1}\right)\right) \rightarrow 0$ as $m, n \rightarrow \infty$. Since $\int_{0}^{1}\left|h_{n}(x)-h_{m}(x)\right|^{2} d \mu(x) \leq \mu\left(\omega\left(h_{n}, h_{m}\right)\right)$ and $\int_{0}^{1}\left|h_{n}^{-1}(x)-h_{m}^{-1}(x)\right|^{2} d \mu(x) \leq \mu\left(\omega\left(h_{n}^{-1}, h_{m}^{-1}\right)\right)$ it follows that $h_{n}$ and $h_{n}^{-1}$ are Cauchy sequences in $L^{2}([0,1))$. Let $f, g$ be their limits in $L^{2}([0,1))$. Passing to a subsequence we may assume that these sequences are pointwise convergent a.e. $([\mu])$. For each $n$ let $[0,1):=U_{n} \cup V_{n}$ be the decomposition (partition) which corresponds to the way we defined the maps $u_{n}$ and $v_{n}$ as in the Theorem 3.3 for the wavelet 
induced map $h_{n}$. In other words, $u_{n}(x)=h_{n}(x)$ for $x \in U_{n}$ and $v_{n}(y)=h_{n}^{-1}(y)$ for $y \in h\left(V_{n}\right)$. We remind the reader that $u_{n}$ and $v_{n}$ can be extended in order that $u_{n} \in \mathcal{W} \mathcal{I}_{1}$ and $v_{n} \in \mathcal{W I}_{2}$.

For $m>n$ we observe that $\left(U_{n} \nabla U_{m}\right) \backslash \mathcal{F} \subset \omega\left(h_{n}, h_{m}\right)$ where $\mathcal{F}$ was defined by (7) in the proof of Proposition 2.3. Indeed, for instance if $x \in\left(U_{n} \backslash U_{m}\right) \backslash \mathcal{F}=U_{n} \cap V_{m}$ it follows that $h_{n}(x)=u_{n}(x)$ and $h_{m}(x)=v_{m}^{-1}(x)$. Since $x \notin \mathcal{F}$ we see that $h_{n}(x) \neq h_{m}(x)$. Hence $\mu\left(U_{n} \nabla U_{m}\right) \rightarrow 0$ as $m, n \rightarrow \infty$. Passing again to a subsequence if necessary we may assume without loss of generality that $\chi_{U_{n}} \rightarrow \chi_{U}$ pointwise for some measurable subset $U$ of $[0,1)$. It follows then that $\mu\left(U_{n} \nabla U\right) \rightarrow 0, \mu\left(V_{n} \nabla V\right) \rightarrow 0$ as $n \rightarrow \infty$ and $\chi_{V_{n}} \rightarrow \chi_{V}$ pointwise where $V=[0,1) \backslash U$. It is clear that $U$ may be empty in which case we will show that $f=g=i d$. If $\mu(U)>0$ then $\chi_{U_{n}}(x) \rightarrow \chi_{U}(x)=1$ for almost every $x \in U$. For such an $x \in U$ we deduce that for some $n_{0}(x) \in \mathbf{N}$ if $n \geq n_{0}(x)$ it follows that $x \in U_{n}$. Hence $h_{n}(x)=u_{n}(x)=\left\lfloor x / 2^{k(x, n)}\right\rfloor \rightarrow f(x)$ or $h_{n}(x)=u_{n}(x)=\left\lfloor(x-1) / 2^{k(x, n)}\right\rfloor \rightarrow f(x)$ with $k(x, n) \in \mathbf{N}$. There are only two possibilities: $k(n, x) \rightarrow \infty$ or $k(n, x)$ is eventually constant. We claim that for almost every $x \in U$ the sequence $k(x, n)$ is eventually constant.

Indeed, suppose that for some subset of $U$ of positive measure, say $\Upsilon$, we have $k(x, n) \rightarrow \infty$ for all $x \in \Upsilon$. Then if we fix $n$ but keep it arbitrary it is clear from our assumption on $\Upsilon$ that $\Upsilon=\bigcup_{m}\{x \in \Upsilon: k(x, m)>k(x, n)\}$. Using the continuity of the Lebesgue measure we may replace $\Upsilon$ with $\Upsilon_{m, n}=\{x \in \Upsilon: k(x, m)>k(x, n)\}$ for some $m$ such that $\mu\left(\Upsilon_{m, n}\right)>\mu(\Upsilon) / 2$. Then it easy to see that $\Upsilon_{m, n} \subset \omega\left(h_{n}, h_{m}\right)$ which implies $0<\mu(\Upsilon) / 2<\mu\left(\omega\left(h_{n}, h_{m}\right)\right)$. This contradicts the fact $\mu\left(\omega\left(h_{n}, h_{m}\right)\right) \rightarrow 0$ and our claimed is proved.

We proved that for almost every $x \in U$ it follows that $k(x, n)$ is an eventually constant sequence. For such an $x$ we let $k(x)=\lim _{n \rightarrow \infty} k(x, n) \in \mathbf{N}$. Then $f(x)=\lim h_{n}(x)=$ $u_{n}(x)=\left\lfloor x / 2^{k(x)}\right\rfloor$ or $f(x)=\left\lfloor(x-1) / 2^{k(x)}\right\rfloor$ depending upon $x \in[1 / 2,1)$ or $x \in[0,1 / 2)$. By Lemma 3.1, $f$ is automatically one-to-one on $U$ and agrees with the characterization of an wavelet induced map in Proposition 2.3.

We consider $U_{n}^{\prime}=h_{n}\left(U_{n}\right)$ and $V_{n}^{\prime}=h_{n}\left(V_{n}\right)$. Since $[0,1)=U_{n} \cup V_{n}$ we get $[0,1)=U_{n}^{\prime} \cup V_{n}^{\prime}$. As before for $m>n$ we observe that $\left(V_{n}^{\prime} \nabla V_{m}^{\prime}\right) \backslash \mathcal{F} \subset \omega\left(h_{n}^{-1}, h_{m}^{-1}\right)$. Then without loss of generality we may assume that $\chi_{V_{n}^{\prime}} \rightarrow \chi_{V^{\prime}}$ pointwise for some measurable subset $V^{\prime}$ of $[0,1)$. As a result $\chi_{U_{n}^{\prime}} \rightarrow \chi_{U^{\prime}}$ pointwise a.e. where $U^{\prime}=[0,1) \backslash V^{\prime}$. For a.e. $x \in U$ we proved that there exist an $n_{1}(x) \in \mathbf{N}$ such that if $n>n_{1}(x)$ we have $f(x)=h_{n}(x)$ and $x \in U_{n}$. Since $h_{n}(x) \in h_{n}\left(U_{n}\right)=U_{n}^{\prime}$ we get $\chi_{U_{n}^{\prime}}(f(x))=1$. Then letting $n \rightarrow \infty$ we get $\chi_{U^{\prime}}(f(x))=1$. This shows that $f(U)=U^{\prime}$ almost everywhere. Therefore if $\mu\left(V^{\prime}\right)=0$ then it must be true that $f(U)=[0,1)$ a.e. $([\mu])$. But this is not possible because $f$ is strictly contractive in the sense of measure. 
Thus, it must be true that $\mu\left(V^{\prime}\right)>0$. As before let us take an $y \in V^{\prime}$ such that $\chi_{V_{n}^{\prime}}(y) \rightarrow$ $\chi_{V^{\prime}}(y)=1$. Thus there exists an $n_{2}(y) \in \mathbf{N}$ such that $y \in V_{n}^{\prime}$ if $n>n_{2}(y)$. By Proposition 2.3 and the construction of $u_{v}, v_{n}$ in Theorem 3.3 we have $h_{n}^{-1}(y)=v_{n}(y)=(y+k(y, n)) / 2^{l(y, n)}$ for some integer $k(y, n)$ and $l(y, n) \in \mathbf{N} \cup\{\mathbf{0}\}$. We now claim that for almost every $y \in V^{\prime}$ the sequence $l(y, n)$ is eventually constant on a subsequence.

Indeed, assuming that for some measurable subset of $V^{\prime}$, say $\Theta$, with $\mu(\Theta)>0$, for $y \in \Theta$ the sequence $l(y, n) \rightarrow \infty$. Then we make the argument as above that for fixed $n$ but arbitrary $\Theta=\bigcup_{m}\{y \in \Theta: l(y, m)>l(y, n)\}$. Hence, there exists $\Theta_{m, n}:=\{x \in \Upsilon$ : $k(x, m)>k(x, n)\}$ for some $m$ such that $\mu\left(\Theta_{m, n}\right)>\mu(\Theta) / 2$. One can check similarly that $\Theta_{m, n} \backslash \mathcal{F} \subset \omega\left(h_{n}^{-1}, h_{m}^{-1}\right)$ which implies $0<\mu(\Theta) / 2<\mu\left(\omega\left(h_{n}^{-1}, h_{m}^{-1}\right)\right)$. This contradicts the fact $\mu\left(\omega\left(h_{n}^{-1}, h_{m}^{-1}\right)\right) \rightarrow 0$ and our claimed is proved.

Using this claim and the fact that $h_{n}^{-1}(y)$ is convergent for almost every $y$ to $g(y)$ we see that the sequence $k(y, n)$ must be eventually constant for a subsequence on which $l(y, n)$ is eventually constant. Letting $n \rightarrow \infty$ we obtain that $g(y)=(y+k(y)) / 2^{l(y)}$ for some $k(y), l(y) \in \mathbf{N} \cup\{\mathbf{0}\}$. This shows that $g$ is in $\mathcal{W I}_{2}$. As we argued before $g\left(V^{\prime}\right)=V$. If $\mu(U)=0$ then $\mu\left(U^{\prime}\right)=0$ and then $g$ must be the identity since it is contractive in the sense of measure.

To finish the prove we extend $f$ from $U$ to $[0,1)$ and $g$ from $V$ to $[0,1)$ so that the new maps $\widetilde{f}$ and $\widetilde{g}$ satisfy: $\widetilde{f} \in \mathcal{W I}_{1}$ and $\widetilde{g} \in \mathcal{W I}_{2}$. Denote $h=\widetilde{f} \diamond \widetilde{g}$. Finally an argument based on the continuity of the Lebesgue measure and the relation (10) shows that $h_{n}$ converges to $h$.

Remark. The proof of Theorem 3.8 although quite lengthy it reveals the understanding of what the convergence in the distance (33) means at the level of wavelet sets and equivalently on $\mathcal{W I}$. 


\section{EXAMPLES}

Consider the Journe wavelet set $J:=\left[-\frac{32 \pi}{7},-4 \pi\right) \cup\left[-\pi,-\frac{4 \pi}{7}\right) \cup\left[\frac{4 \pi}{7}, \pi\right) \cup\left[4 \pi, \frac{32 \pi}{7}\right)$. Then its wavelet induced function denoted by $\widetilde{h}_{J}$ can be described by

$$
\widetilde{h}_{J}(x)= \begin{cases}\left\lfloor 2^{-1}(x-1)\right\rfloor & \text { on } \quad\left[0, \frac{3}{7}\right), \\ \left\lfloor 2^{2} x\right\rfloor & \text { on }\left[\frac{3}{7}, \frac{4}{7}\right), \\ \left\lfloor 2^{-1} x\right\rfloor & \text { on }\left[\frac{4}{7}, 1\right) .\end{cases}
$$

In this case one can choose $u \in \mathcal{W I}_{1}$ defined by

$$
u(x)= \begin{cases}\left\lfloor 2^{-1}(x-1)\right\rfloor & \text { on } \quad\left[0, \frac{1}{2}\right), \\ \left\lfloor 2^{-1} x\right\rfloor \text { on }\left[\frac{1}{2}, 1\right),\end{cases}
$$

and $v \in \mathcal{W I}_{2}$ defined by

$$
v(x)=\left\{\begin{array}{lll}
\frac{x+2}{4} & \text { on } & {\left[0, \frac{1}{2}\right)} \\
\frac{x+1}{4} & \text { on } & {\left[\frac{1}{2}, 1\right)}
\end{array}\right.
$$

such that $\widetilde{h}_{J}=u \diamond v$.

Let us go the other way in our construction. Arguably, the simplest map $u \in \mathcal{W} \mathcal{I}_{1}$ that one can take is given in (19) and we pick one of the simplest maps $v \in \mathcal{W I}_{2}$ defined by $v(x)=x / 2$ for all $x \in[0,1)$. Then the wavelet induced map, $h=u \diamond v$, that is obtained by 
the Schröder-Cantor-Bernstein construction is defined by

$$
h(x)=\left\{\begin{array}{l}
\frac{x}{2} \text { on } \quad\left[\frac{1}{2}, 1\right), \\
2 x \text { on } \quad\left[\frac{1}{3}, \frac{1}{2}\right), \\
\frac{x+1}{2} \text { on } A, \\
2 x \text { on }\left[0, \frac{1}{3}\right) \backslash A,
\end{array}\right.
$$

where $A=\bigcup_{n=1}^{\infty}\left[z_{n}, x_{n+1}\right)$ and the sequences $\left\{z_{n}\right\}_{n \geq 1},\left\{x_{n}\right\}_{n \geq 1}$ are given by the formulae $z_{n}=$ $\frac{1}{3}\left(1-\frac{5}{2 \times 4^{n}}\right), x_{n}=\frac{1}{3}\left(1-\frac{1}{4^{n-1}}\right)$. This will give rise to a wavelet set containing infinitely many intervals $W:=\left[\frac{\pi}{2}, \pi\right) \cup\left[\frac{-8 \pi}{3},-2 \pi\right) \cup(\pi A-\pi) \cup\left(\left[-4 \pi, \frac{-8 \pi}{3}\right) \backslash(4 \pi A-4 \pi)\right)$. It is interesting to mention that if one modifies $v$, as in the proof of Theorem 3.5. to $v(x)=x / 2$ for $x \in[0,2 / 7)$ and $v(x)=x$ if $x \in[2 / 7,1)$ then the wavelet set constructed from $u$ and the new $v$ has only six intervals.

Acknowledgments. First I would like to express my thanks to Professor David Larson for introducing me to the subject of wavelet theory years ago, and for his encouragements that determined me to pursue this research. Proposition 2.3 appears just with statement (parts (i)-(iii)) in [2] and the seed of Theorem 3.3 was the subject of discussions with Professor E. Azoff while I was a postdoctoral associate at University of Georgia, Athens. Hence I am greatly thankful to Professor Azoff for his collaboration and continuous support during a period of more than five years. Last but not least I am thankful to the refree who had the patience to carefully read a previous version of this paper and who pointed out to me how to significantly improve the presentation of the material. 


\section{REFERENCES}

[1] E. Azoff and E. Ionascu, Wandering sets for a class of Borel isomorphisms of [0, 1), Journal of Fourier Analysis and Applications, Vol. 6, 2002

[2] E. Azoff, E. Ionascu, D. Larson and C. Pearcy, Direct paths of wavelets, to appear in Houston Journal of Math.

[3] L. Baggett, H. Madina and K. Merrill, Generalized multiresolution analysis and a construction procedure for all wavelet sets in $\mathbb{R}^{n}$, J. Fourier Analysis and Applications, 5(1999), 563-573.

[4] X. Dai, Y. Diao and Q. Gu, Frame Wavelet Sets in R, Proceedings of the AMS, vol. 129, 7(2000), 2045-2055.

[5] X. Dai, Y. Diao and Q. Gu, Subspaces with normalized tight frame wavelets in $\mathbf{R}$, Proceedings of the AMS, vol. 130, 6(2002), 1661-1667.

[6] X. Dai, Y. Diao, Q. Gu and D. Han, Frame wavelet sets in $\mathbf{R}^{\mathbf{d}}$, to appear in J. Comput. Appl. Math..

[7] X. Dai, Y. Diao, Q. Gu and D. Han, Wavelets with frame multiresolution analysis, to appear in J. Fourier Analysis and Applications.

[8] X. Dai, Y. Diao, Q. Gu and D. Han, The existence of subspace wavelet sets, to appear in J. Comput. Appl. Math..

[9] X. Dai and D. Larson, Wandering vectors for unitary systems and orthogonal wavelets, Memoirs Amer. Math. Soc.,134(1998).

[10] X. Dai, D. Larson and D. Speegle, Wavelets in $\mathbb{R}^{n}$.I., J. Fourier Anal. Appl. 3(1997), 451-456.

[11] X. Dai, D. Larson and M. Speegle, Wavelet sets in $\mathbb{R}^{n}$. II., Wavelets, multiwavelets, and their applications (San Diego, CA, 199\%), 15-40, Contemp. Math., 216, Amer. Math. Soc., Providence, RI, 1998.

[12] X. Fang and X. Wang, Construction of minimally-supported-frequency wavelets, J. Fourier Anal. and Appl. 2(1996), 315-327.

[13] G. Garrigos and D. Speegle, Completeness in the set of wavelets, Proc. Amer. Math. Soc. 128 (2000), no. 4, 1157-1166.

[14] E. Hernandez, X. Wang and G. Weiss, Smoothing minimally supported frequency wavelets. I., J. Fourier Anal. Appl. 2(1996), 329-340.

[15] E. Hernandez, X. Wang and G. Weiss, Smoothing minimally supported frequency wavelets. II., J. Fourier Anal. Appl. 3(1997), 23-41.

[16] E. Ionascu, D. Larson and C. Pearcy, On wavelets sets, J. Fourier Analysis and Applications, J. Funct. Anal., 157 (1998), 413-431.

[17] E. Ionascu, D. Larson, and C. Pearcy, On the unitary systems affiliated with orthonormal wavelet theory in n-dimensions, J. Funct. Anal., 157(1998), 413-431.

[18] E. Ionascu and C. Pearcy, On subwavelet sets, Proc. Amer. Math. Soc. 126( 1998), 3549-3552.

[19] A. Rényi, Representations for real numbers and their ergodic properties, (English) Acta Math. Acad. Sci. Hungar, (1957), 477-493.

[20] D. Speegle, The s-elementary wavelets are path-connected, Proc. Amer. Math. Soc., 127 (1999), no. 1, 223-233. MR 99b:42045

Current address: Columbus State University, 4225 University Avenue, Columbus, GA 31907

E-mail address: ionascu_eugen@ colstate.edu 